\title{
Pretibial myxedema in a euthyroid patient without history of autoimmune thyroid disease
}

\author{
Panagiotis Anagnostis ${ }^{1}$, Aikaterini Artzouchaltzi ${ }^{1}$, Alexandra Grekou ${ }^{2}$, Dimitrios Stogiannou ${ }^{1}$, Anastasia \\ Skalkou ${ }^{1}$, Nifon Gkekas ${ }^{1}$, Spyridon Gerou ${ }^{3}$, Vasilios Tzalokostas ${ }^{1}$
}

\begin{abstract}
1Divisions of Dermatology and Endocrinology, Police Medical Center of Thessaloniki, Thessaloniki, Greece 2Laboratory of Histopathology "Histodiagnostiki", Thessaloniki, Greece

3Laboratories "Analysis", Thessaloniki, Greece
\end{abstract}

Introduction: Pretibial myxedema (PM) is a rare extrathyroidal manifestation of Graves' disease (GD), in about 1-5\% of patients, usually co-existing with Graves' orbitopathy. PM constitutes one of the classical forms of dermopathy in GD, presenting with a variety of symptomatology (from asymptomatic erythema to severe elephantiasic form). PM usually occurs during the hyperthyroid state of GD. PM has also rarely been described in patients with Hashimoto thyroiditis.

Case report: A 61-year old Caucasian woman attended our outpatient dermatology clinic for evaluation and management of itchy bilateral erythema, involving the lower two-thirds of the pretibial regions. This had also appeared 11 years ago.

Her medical history was remarkable for subtotal thyroidectomy due to non-toxic multinodular goiter 15 years ago. There was no history of thyrotoxicosis or ophthalmopathy. The medical history also included breast cancer, type 2 diabetes mellitus (T2DM) and arterial hypertension. Her family history was positive for T2DM, breast and renal carcinoma. The patient was a non-smoker and did not consume any alcohol. Except for thyroxine, the patient was also on letrozole, sitagliptin/metformin, glimepiride, olmesartan and moxonidine.

Clinical examination revealed the presence of bilateral, edematous pretibial erythema, with "peau d' orange" appearance. No signs of ophthalmopathy or acropathy were observed.

Laboratory investigation showed the following findings: thyroid stimulating hormone (TSH): 0.89 mIU/L (normal range: 0.4-4) and free-thyroxine: $1.32 \mathrm{ng} / \mathrm{mL}$ (normal range: 0.8-1.5). Thyroid peroxidase (TPO-Ab) and thyroglobulin auto-antibodies (Tg-Ab) were negative, as were the levels of thyroid stimulating immunoglobulins (TSI) and thyroid binding inhibitory immunoglobulins (TBII). The rest of laboratory investigation was unremarkable, except for vitamin D deficiency (25-hydroxy-vitamin D concentrations: 17 $\mathrm{ng} / \mathrm{mL}$, normal values $>30 \mathrm{ng} / \mathrm{mL}$ ). The patient's glycaemic control was satisfactory (HbA1c: $5.8 \%$ ).

Neck ultrasonography revealed the presence of significant thyroid remnant, with mild heterogeneous echogenity, without nodules.

Due to the non-typical manifestation of the skin lesion (i.e. absence of clinical and laboratory evidence of thyroid autoimmune disease), a skin biopsy was performed, which was consistent with PM. In detail, it showed edema of the reticular dermis as empty slit-like spaces, separating collagen fibers, with normal fibroblasts. Stellate fibroblasts (myofibroblasts) in papillary dermis and perivascular infiltrate of lymphocytes were also observed. The histochemical stain Alcian blue pH 2.5 showed mild increase of acid mucopolysaccharides in papillary dermis (Fig. 1).

The patient was successfully managed with topical application of corticosteroids, showing significant amelioration of her lesions one month later, sustained 12 months after therapy (Fig. 2).

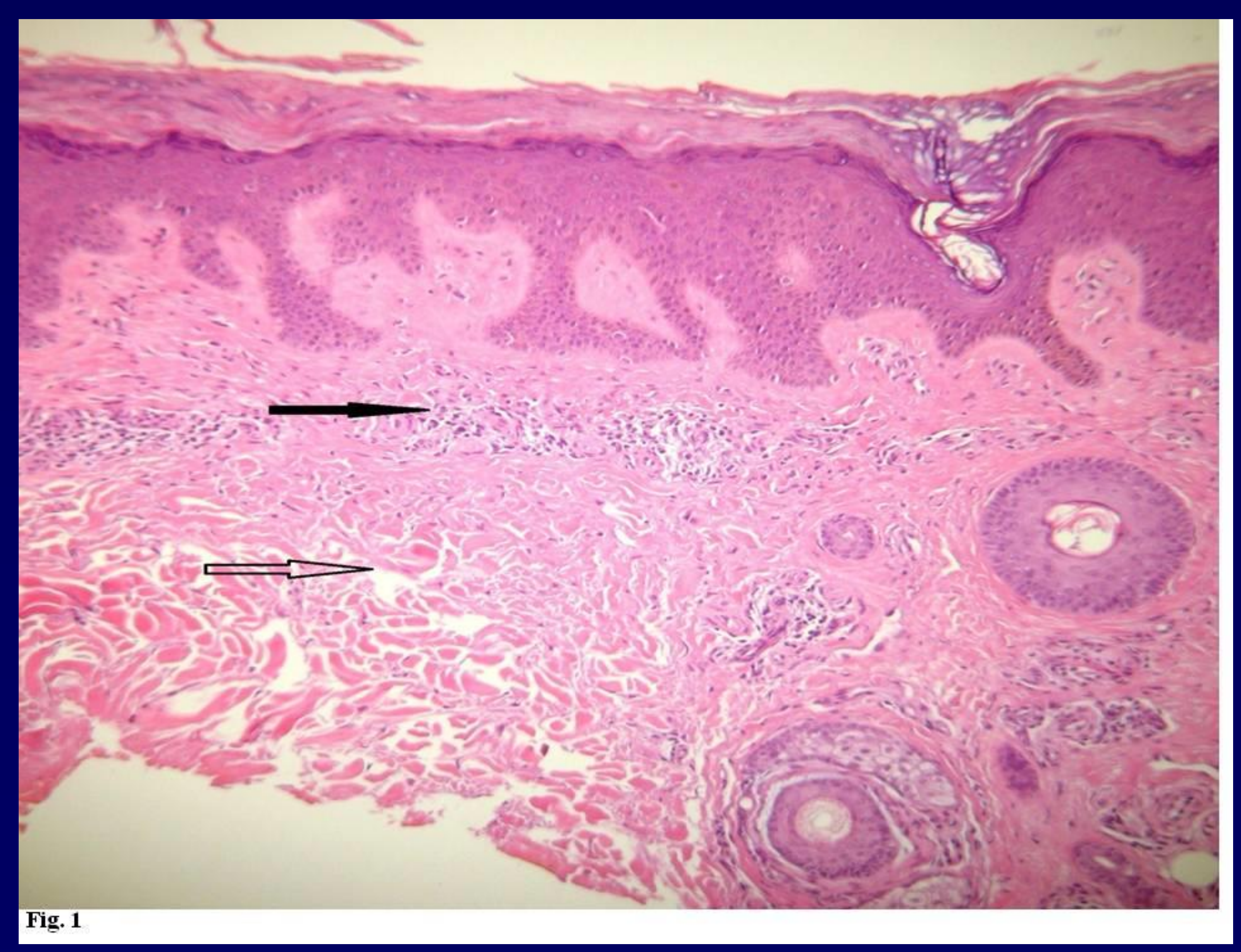

Fig. 1

Skin biopsy showing edema of the reticular dermis as empty slit like spaces between collagen fibers (empty arrow) and perivascular lymphocytic infiltrates (black arrow) (H\&EX200).

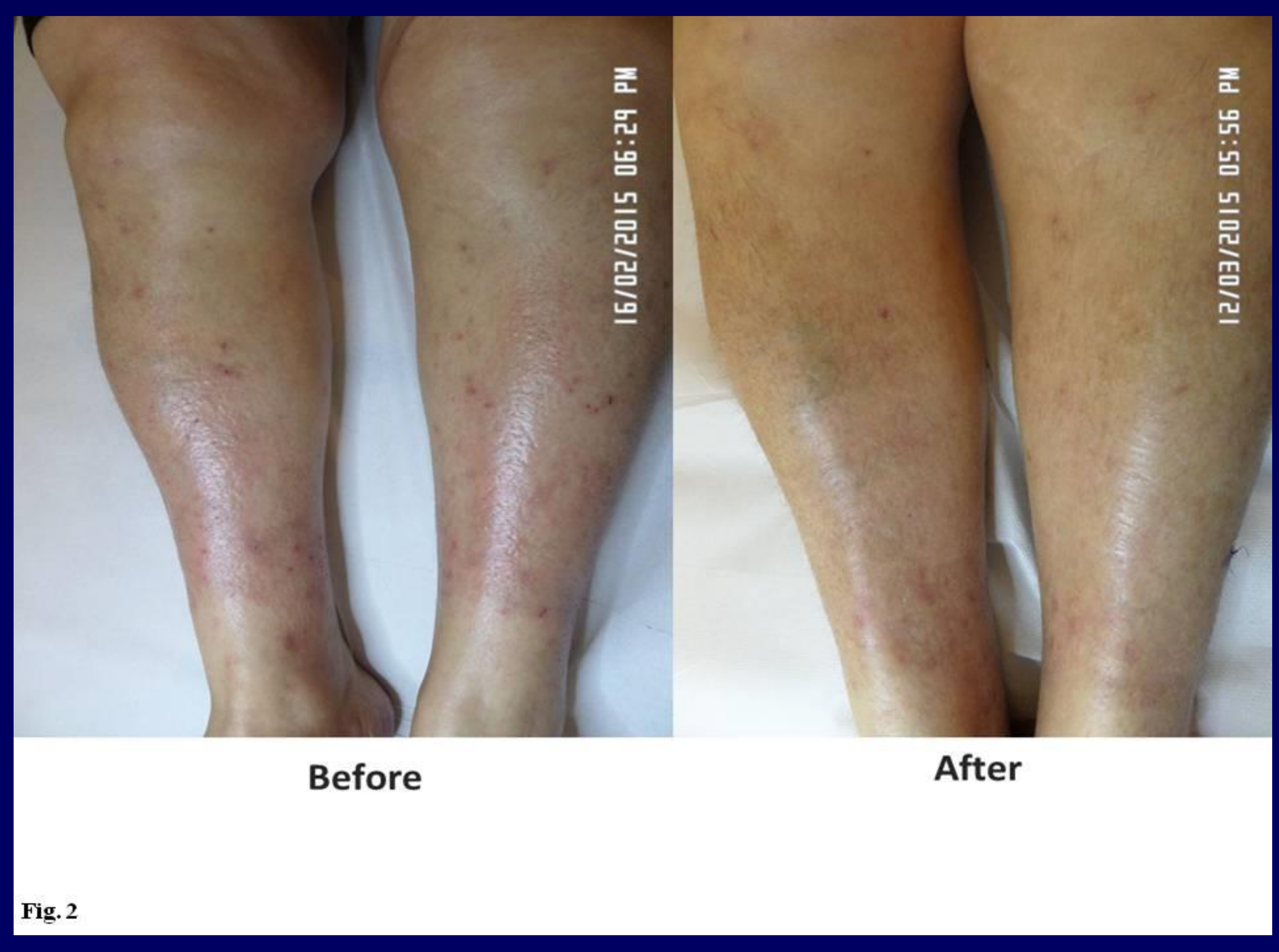

Fig. 2

Skin lesions before and after successful corticosteroid application

Conclusions: This is a rare case of PM in a euthyroid patient, with no evidence of thyroid autoimmunity. Six other euthyroid PM cases have been reported (one with positive TSI, two with negative TSI and not measured in the other cases).

References:

1. Anagnostis P, Adamidou F, Polyzos SA, Katergari S, Karathanasi E, Zouli C, Panagiotou A, Kita M. Predictors of long-term remission in patients with Graves' disease: a single center experience. Endocrine 44:448-453, 2013.

2. Anagnostis P, Adamidou F, Poulasouchidou M, Karras S. Severe eyelid oedema in Graves' ophthalmopathy. BMJ Case Rep Jul 13, 2013.

3. Fatourechi V. Pretibial myxedema: pathophysiology and treatment options. Am J Clin Dermatol 6:295-309, 2005.

4. Nair PA, Mishra A, Chaudhary A. Pretibial Myxedema Associated with Euthyroid Hashimoto's Thyroiditis: A Case Report. J Clin Diagn Res 8:YD01-YD02, 2014. 